\title{
DIFERENCIAS DE GÉNERO EN EL AFRONTAMIENTO EN LA ADOLESCENCIA
}

\author{
Gema Martín Seoane \\ Universidad Complutense de Madrid \\ Beatriz Lucas Molina \\ Universidad de La Rioja \\ Rosa Pulido Valero \\ Universidad Complutense de Madrid
}

\begin{abstract}
RESUMEN: En este estudio se analizan las diferencias de género en las estrategias que utilizan los y las adolescentes para afrontar los problemas de la vida cotidiana. Con este fin se seleccionó una muestra representativa de 1.715 estudiantes escolarizados, de edades comprendidas entre 14 y 18 años de la Comunidad de Madrid. Para evaluar el afrontamiento emocional se utilizó la Escala de Afrontamiento para Adolescentes (A.C.S.) elaborada por Frydenberg y Lewis (1997). Los resultados apuntan a un mayor repertorio de estrategias en el caso de las adolescentes, así como diferencias en los estilos de afrontamiento, establecidos a través del análisis factorial de las 18 estrategias de afrontamiento evaluadas.
\end{abstract}

Palabras clave: Género, afrontamiento, adolescencia.

\section{GENDER DIFFERENCES IN COPING DURING ADOLESCENCE}

ABSTRACT: This paper reports the differences between boys and girls in the way they cope with everyday problems. A representative sample of 1,715 students from Madrid was selected. Coping strategies were evaluated by using the Adolescents Coping Scale -ACS- (Frydenberg \& Lewis, 1997). The results showed that girls use a great variety of coping strategies and also differences in coping styles obtained through the factor analysis conducted over the 18 strategies.

Keywords: Gender, coping, adolescence. 
La adolescencia constituye una etapa evolutiva de transición a la vida adulta, caracterizada por la vivencia de continuos cambios a nivel físico, emocional, cognitivo y social que pueden convertirse en una fuente de estrés. Cómo afrontan los y las adolescentes estos cambios y las repercusiones que estos generan en su desarrollo psicosocial, ha sido una de las líneas de investigación más ampliamente estudiadas (Bermúdez, Teva y Buela-Casal, 2009; Donenberg y Pao, 2005; Frydenberg, 1994; Hampel y Peterman, 2006).

En este sentido, se presta una especial atención a las funciones que cumple el afrontamiento (Lazarus y Folkman, 1984): a) Modificar la situación o acontecimiento estresante a través de la utilización de estrategias focalizadas en el problema; entre las que se incluirían aquellas destinadas a alterar la relación descompensada entre la persona y su entorno, mediante la modificación de las circunstancias problemáticas o mediante la aportación de nuevos recursos que contrarresten el efecto negativo de las condiciones ambientales; y b) Regular los estados emocionales evocados por la situación o acontecimiento estresante a través de la utilización de estrategias centradas en la emoción, como la evitación o la búsqueda de apoyo social. Estos trabajos de Lazarus y Folkman han generado una amplia línea de investigación centrada en el análisis de los tipos de afrontamiento y en la elaboración de instrumentos (Fanshawe y Burnett, 1991; Patterson y McCubbin, 1987; Spirito, Stark y Williams, 1988).

En la evaluación del afrontamiento en adolescentes destacan los trabajos de Frydenberg y Lewis (1996), que a partir del estudio de las conductas de afrontamiento más frecuentes de los adolescentes, elaboraron una escala en la que se incluyen dieciocho estrategias de afrontamiento que se distribuyen entre los dos estilos propuestos anteriormente por Lazarus y Folkman dirigido a la resolución del problema y afrontamiento en relación con los demás, y un tercer estilo, el improductivo, propuesto por Frydenberg y Lewis (1996).

Los resultados de las investigaciones sobre las estrategias de afrontamiento en función del género en adolescentes no son concluyentes (Mullis y Chapman, 2000). Frydenberg y Lewis (1991), en sus primeros trabajos, señalaron la ausencia de diferencias, sin embargo posteriormente (Frydenberg y Lewis, 1999) observaron que los varones utilizaban la búsqueda de diversiones relajantes y la distracción física más que las mujeres (Recklitis y Noam, 1999). En las estrategias vinculadas a un estilo de afrontamiento focalizado en la emoción parece haber un mayor acuerdo, relacionándose con las mujeres adolescentes en la búsqueda de apoyo social (Frydenberg y Lewis, 1991; Frydenberg y Lewis, 1999; Plancherel y Bolognini, 1995; Plancherel, Bolognini y Halfon, 1998; Parsons, Frydenberg y Poole, 1996; Recklitis y Noam, 1999; Washburn, Hillman, Sawilowsky, 2004). Por otra parte, en un estudio, realizado por González, Montoya, Casullo y Bernabeu (2002), encuentran que las adolescentes contaban con un mayor repertorio de estrategias de afrontamiento, aunque parecían ser menos habilidosas para afrontar situaciones problemáticas en comparación con los varones. 
En este contexto, el presente estudio pretende avanzar en el análisis de las diferencias de género en los estilos y estrategias de afrontamiento de los y las adolescentes.

\section{Método}

\section{Participantes}

Los participantes fueron 1.715 adolescentes escolarizados en centro educativos de la Comunidad de Madrid y de edades comprendidas entre 14 y 18 años, siendo el porcentaje de varones de un 53\% y un $47 \%$ el de las mujeres. La media de edad para las mujeres es de 16,08 con una desviación típica de 1,49 , en el caso de los hombres los valores resultaban muy parecidos, siendo la media de edad 16,10 con una desviación típica de 1,41. Para garantizar la equivalencia del género en cuanto a la edad se realizó una prueba de diferencia de medias y el valor $\mathrm{F}$ resultante de comparar estas medias fue de 0,117, no significativo a un nivel de probabilidad del 5\%. La selección de los participantes se realizó mediante un muestreo aleatorio por conglomerados, siendo la unidad de análisis el centro educativo.

\section{Instrumento de medida}

Para evaluar los estilos y estrategias de afrontamiento se utilizó la versión española de la Escala de Afrontamiento para Adolescentes (Frydenberg y Lewis, 1997). La consistencia interna de la adaptación española es de $\alpha=0,76$ con un coeficiente de fiabilidad test-retest de $r x y=0,68$. El ACS es un inventario de autoinforme compuesto de 80 elementos, 79 de tipo cerrado y uno final abierto, que permite evaluar con fiabilidad 18 estrategias de afrontamiento diferentes. Los 79 elementos cerrados se puntúan mediante una escala tipo Likert de 1 a 5: 1. No me ocurre nunca o no lo hago; 2. Me ocurre o lo hago raras veces; 3. Me ocurre o lo hago algunas veces; 4. Me ocurre o lo hago a menudo; 5 . Me ocurre o lo hago con mucha frecuencia. Aunque este instrumento presenta dos formas (una específica y otra general), en esta investigación se ha utilizado la Forma General, que mide las estrategias de afrontamiento que un sujeto suele elegir con independencia de la naturaleza del problema, es decir, la manera habitual de afrontar las situaciones que le desbordan.

El cuestionario ACS consta de 18 escalas que se corresponden con las diferentes estrategias de afrontamiento: Buscar apoyo social, Concentrarse en resolver el problema, Esforzarse y tener éxito, Preocuparse, Invertir en amigos íntimos, Buscar pertenencia, Hacerse ilusiones, Falta de afrontamiento, Reducción de la tensión, Acción social, Ignorar el problema, Autoinculparse, Reservarlo para sí, Buscar apoyo espiritual, Fijarse en lo positivo, Buscar ayuda profesional, Buscar diversiones relajantes, Distracción física. Según los autores, estas estrategias pueden agruparse en tres estilos básicos de afrontamiento: Di- 
rigido a la resolución del problema, Afrontamiento en relación con los demás y Afrontamiento improductivo.

\section{Procedimiento}

La evaluación fue realizada por el grupo de investigadoras, autoras de este trabajo. Se solicitó la colaboración del centro educativo mediante una carta dirigida a la dirección en la que se explicaban los aspectos generales del estudio, así como el compromiso del equipo de investigación de devolución de los datos a través de un informe. La aplicación del instrumento fue colectiva, desarrollándose en el centro educativo en horario escolar. La participación fue de carácter voluntario, aunque ningún estudiante se negó a participar.

\section{Resultados}

Sobre los estilos y estrategias de afrontamiento

De las 18 estrategias evaluadas, las estrategias que presentan una media más elevada son buscar diversiones relajantes $(78,75)$, preocuparse $(73,31)$, invertir en amigos íntimos $(72,42)$ y buscar pertenencia $(72,24)$. En el polo opuesto, las estrategias con las medias más bajas son acción social $(40,55)$, reducción de la tensión $(41,61)$, ignorar el problema $(42,58)$ y buscar apoyo espiritual $(42,98)$.

Para establecer los estilos de afrontamiento, a partir de las 18 estrategias, se realizó un análisis factorial para reducir el conjunto a un número menor de factores, se examinaron varias soluciones con distinto número de componentes según la aproximación del Scree test (de 4 a 6 factores), optando finalmente por la solución de 5 . El conjunto de los 5 factores explica el $63 \%$ de la varianza total. Los cinco factores resultantes podrían interpretarse como:

Factor 1: Afrontamiento negativo, denominado así por su relación estrecha con la indefensión y con un estado emocional de angustia o culpa, incluye las siguientes estrategias: reservarlo para sí, falta de afrontamiento, autoinculparse, ignorar el problema, reducción de la tensión y hacerse ilusiones. Ejemplos de elementos que configuran las estrategias de este factor: "Ilorar o gritar", "buscar una forma de aliviar la tensión (llorar, gritar, beber, tomar drogas....", no puedo hacer nada para resolver el problema, así que no hago nada", "sentirme culpable", "criticarme a mí mismo", "me pongo malo".

Factor 2: Afrontamiento a través del grupo, denominado así por estar centrado en la búsqueda de apoyo a través del grupo de iguales y de las personas del entorno más próximo del adolescente, incluye las siguientes estrategias: invertir en amigos íntimos, buscar pertenencia, buscar diversiones relajantes y buscar apoyo social. Ejemplos de elementos del cuestionario: "salir y divertirme para olvidar mis dificultades", "conseguir apoyo de mis padres y amigos", "reunirme con amigos", "buscar tiempo para actividades de ocio". 
Factor 3: Afrontamiento constructivo, encaminado a la resolución activa del problema, incluye las siguientes estrategias: esforzarse y tener éxito, preocuparse, concentrarse en resolver el problema. Ejemplos de elementos del cuestionario: "pensar distintas formas de resolver el problema", "considerar otros puntos y tenerlos en cuenta", "dedicarme a resolver el problema poniendo en juego todas las posibilidades", "dedicarme a resolver lo que provoca el problema".

Factor 4: Ayuda externa, denominada así para referirse a un afrontamiento a través de búsqueda de ayuda fuera del entorno personal. Incluye estrategias relacionadas con la búsqueda de ayuda especializada, como pedir ayuda profesional, ayuda espiritual y acción social. Ejemplos de elementos de este factor: "ir a reuniones en las que se estudia el problema", "leer un libro sagrado o de religión", "pedir ayuda profesional".

Factor 5: Afrontamiento basado en la distracción. Incluye las estrategias distracción física y fijarse en lo positivo. Ejemplos de elementos de este factor: "tratar de tener una visión alegre de la vida", "hacer deporte", "mantenerme en forma y con buena salud".

En la siguiente tabla se presenta la matriz factorial rotada con las saturaciones de las estrategias en cada factor.

TABLA 1. Matriz factorial rotada. Rotación Varimax

\begin{tabular}{|l|c|c|c|c|c|}
\hline \multirow{2}{*}{ Estrategias } & \multicolumn{5}{c|}{ Componente } \\
\cline { 2 - 6 } & $\mathbf{1 1}$ & $\mathbf{2 2}$ & $\mathbf{3 3}$ & $\mathbf{4 4}$ & $\mathbf{5 5}$ \\
\hline Reservarlo para sí &., 764 & & & & \\
\hline Falta de afrontamiento &., 724 & & & & \\
\hline Autoculparse &., 699 & & & & \\
\hline Ignorar el problema &., 583 & & & &., 406 \\
\hline Reducción de la tensión &., 568 &., 406 & & &,- 313 \\
\hline Hacerse ilusiones &., 467 &., 392 & & & \\
\hline Invertir en amigos íntimos & &., 775 & & & \\
\hline Buscar pertenencia & &., 661 &., 393 & & \\
\hline Buscar diversiones relajantes & &., 605 & & &., 486 \\
\hline Buscar apoyo social & &., 563 &., 380 &., 472 & \\
\hline Preocuparse & &., 356 &., 727 & & \\
\hline Esforzarse y tener éxito & & &., 718 & &., 378 \\
\hline Concentrarse en resolver el problema & &., 353 &., 703 &., 263 & \\
\hline Acción social & & & &., 726 & \\
\hline Buscar ayuda profesional & & & &., 726 & \\
\hline Buscar apoyo espiritual & & & &., 678 & \\
\hline Distracción física & & & & &., 664 \\
\hline Fijarse en lo positivo & &., 347 & & &., 506 \\
\hline
\end{tabular}

Método de extracción: Análisis de componentes principales.

Método de rotación: Normalización Varimax con Kaiser.

Se han eliminado las saturaciones inferiores a 0,30 
Las puntuaciones obtenidas en estos factores, revelan que los estilos de afrontamiento más utilizados por los y las adolescentes son en primer lugar el Afrontamiento a través del grupo (media=293,69, DT=45,34), seguido del Afrontamiento Negativo (media=291,26, DT=59,78), el Afrontamiento Constructivo (media=212,26, DT=32,15), la Ayuda externa (media=137,48, $\mathrm{DT}=35,96)$ y, en último lugar, el Afrontamiento a través de la distracción (media=134,95, DT=28,52).

Sobre las diferencias de género en el afrontamiento

A continuación se presentan los resultados encontrados en relación a las diferencias en la utilización de estrategias de afrontamiento en función del "género". Para ello se utilizó el estadístico $t$ de Student.

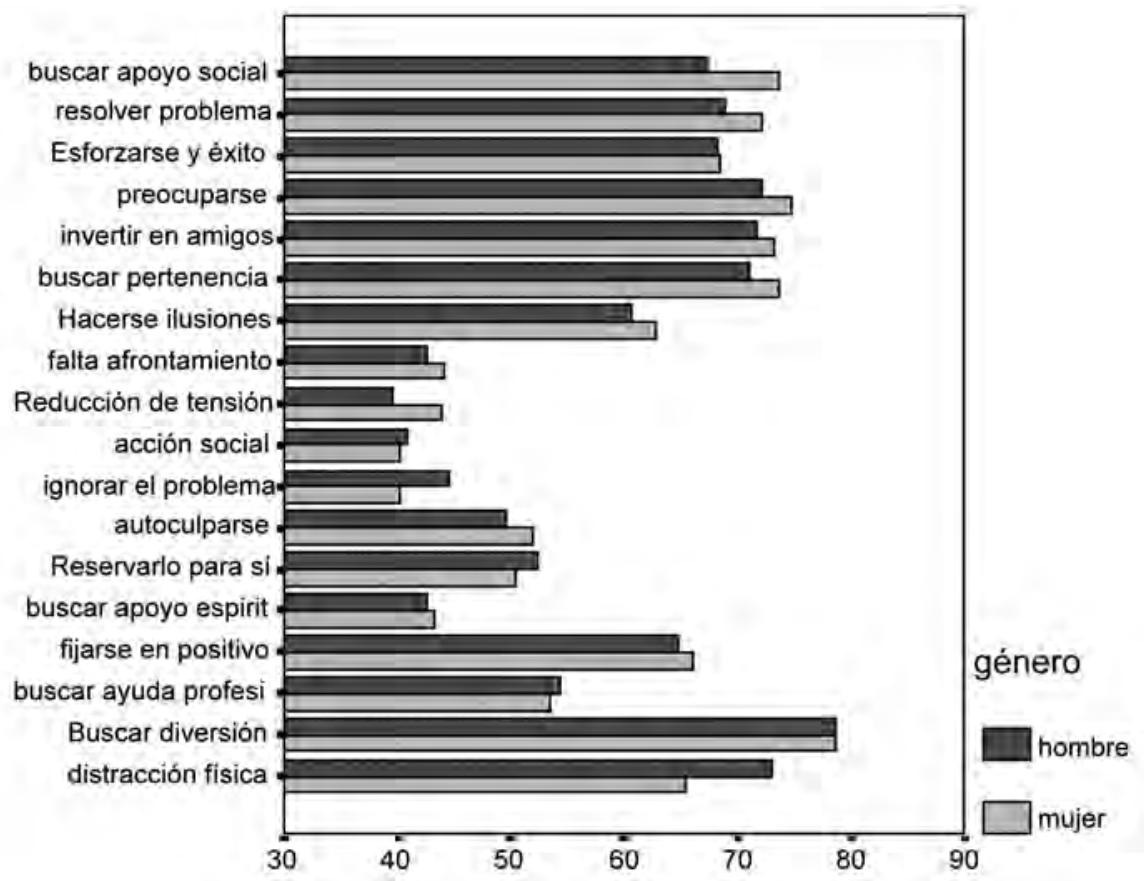

Figura 1. Comparación de medias de las estrategias de afrontamiento en función del género.

Como puede observarse, comparando las medias obtenidas en cada estrategia se encuentran diferencias significativas en las siguientes estrategias: los adolescentes obtienen puntuaciones superiores en ignorar el problema $(\mathrm{t}(1713)=-6,056 ; \mathrm{p}<0,001)$, reservarlo para sí $(\mathrm{t}(1713)=-2,393 ; \mathrm{p}<0,05)$ y distracción física $(\mathrm{t}(1713)=-7,508 ; \mathrm{p}<0,001)$. Las adolescentes destacan de 
forma significativa en: buscar apoyo social $(\mathrm{t}(1713)=8,177 ; \mathrm{p}<0,001)$, concentrarse en resolver el problema $(\mathrm{t}(1713)=5,071 ; \mathrm{p}<0,001)$, preocuparse $\mathrm{t}(1713)=4,170 ; \mathrm{p}<0,001)$, invertir en amigos $(\mathrm{t}(1713)=2,293 ; \mathrm{p}<0,05)$, bus car pertenencia $(\mathrm{t}(1713)=4,317 ; \mathrm{p}<0,001)$, hacerse ilusiones $(\mathrm{t}(1713)=2,894$; $\mathrm{p}<0,01)$, falta de afrontamiento $(\mathrm{t}(1713)=2,121 ; \mathrm{p}<0,05)$, reducción de la tensión $(\mathrm{t}(1713)=6,161 ; \mathrm{p}<0,001)$ y autoculparse $(\mathrm{t}(1713)=3,053 ; \mathrm{p}<0,01)$. No se encuentran diferencias significativas entre los y las adolescentes en las estrategias de esforzarse y tener éxito, acción social, buscar apoyo espiritual, fijarse en lo positivo, buscar ayuda profesional y diversiones relajantes.

Analizando los estilos de afrontamiento, se encuentran diferencias significativas entre chicos y chicas en las siguientes direcciones: las chicas obtienen medias superiores en el factor Afrontamiento a través del grupo $(\mathrm{t}(1713)=4,837$; $\mathrm{p}<0,001)$ y en Afrontamiento constructivo (t(1713)=3,982; $\mathrm{p}<0,001)$, mientras que los chicos obtienen puntuaciones superiores en el factor Afrontamiento a través de la distracción $(\mathrm{t}(1713)=-4,539 ; \mathrm{p}<0,001)$. No se encuentran diferencias significativas en el factor Afrontamiento negativo y en Ayuda externa. En la figura siguiente pueden observarse estas diferencias.

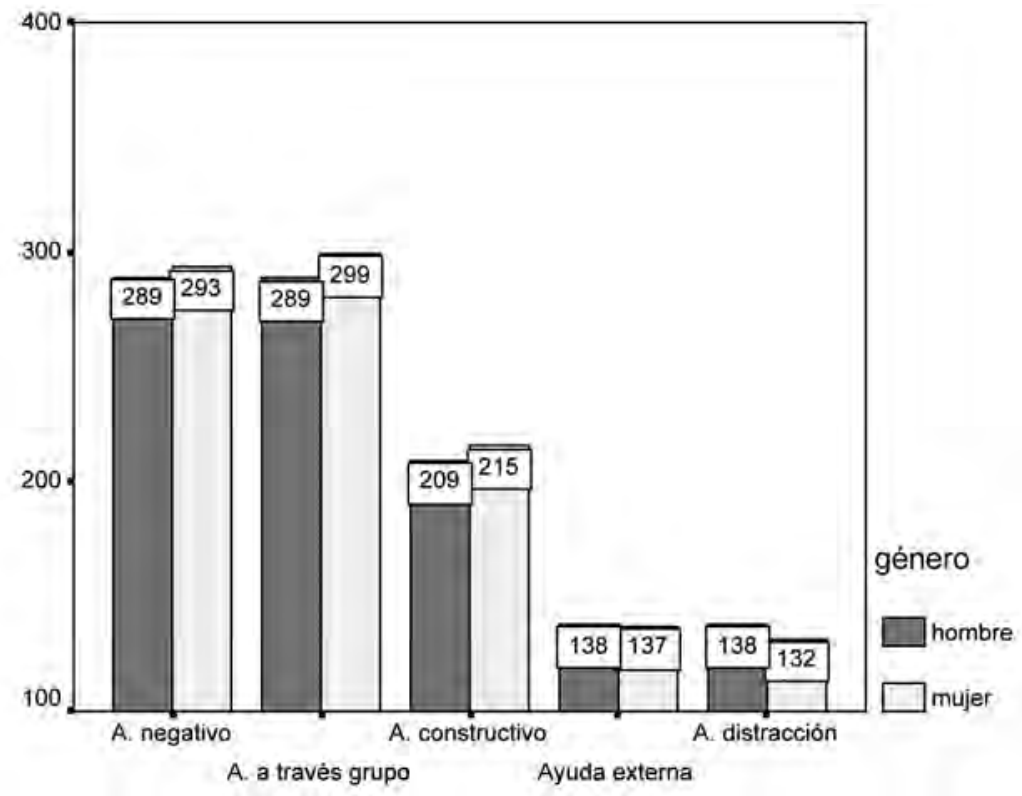

Figura 2. Medias de cada factor en función del género.

\section{Discusión}

Los resultados hallados en este estudio muestran cómo las chicas parecen utilizar en mayor medida estrategias relacionadas con un estilo de afrontamien- 
to a través del grupo, como la búsqueda de apoyo social, invertir en amigos o la búsqueda de pertenencia. Resultado que va en la línea de la mayoría de los estudios previos (Bermúdez, Teva y Buela-Casal, 2009; Frydenberg y Lewis, 1991; González, Montoya, Casullo y Bernabé, 2002; Plancherel y Bolognini, 1995). Es importante destacar que, en contradicción con otros estudios (González, Montoya, Casullo y Bernabé, 2002) las chicas también parecen utilizar en mayor medida estrategias centradas en un estilo de afrontamiento constructivo, como concentrarse en resolver el problema o preocuparse. Sin embargo, siguen utilizando en mayor medida estrategias como autoculparse o la falta de afrontamiento (Galaif, Sussman, Chou y Wills, 2003; Hampel y Peterman, 2006).

En el caso de los chicos los resultados encontrados apuntan a que éstos utilizan en mayor medida un estilo de afrontamiento centrado en la distracción, así como estrategias centradas en ignorar el problema o reservarlo para sí. Estos resultados parecen coincidir con la mayoría de estudios (Bermúdez, Teva y Buela-Casal, 2009; Frydenberg, 2000; González, Montoya, Casullo y Bernabé, 2002).

Estos resultados parecen un reflejo de la situación actual en relación a las diferencias de género: por un lado, los niveles de igualdad alcanzados y la superación de las formas más claras de sexismo parecen estar en la base de esa superioridad de las adolescentes en un afrontamiento constructivo. Sin embargo, los resultados en relación a su superioridad de las chicas en el afrontamiento a través de grupo, como medida de búsqueda de aprobación y apoyo social, y en las estrategias "falta de afrontamiento" o "autoculparse" como se observa en otros estudios (Frydenberg y Lewis, 1999; Frydenberg, 2000) y la superioridad en afrontamiento a través de la distracción y en las estrategias "ignorar el problema y reservarlo para sí" en el caso de los chicos, ponen de manifiesto que aún queda mucho por avanzar en la superación del sexismo. En este sentido, las investigaciones apuntan a la influencia de las pautas de socialización en la forma de afrontar las dificultades. Así los estudios realizados revelan que las adolescentes que construyen su identidad en torno a estereotipos sexistas femeninos utilizan en mayor medida las formas de afrontamiento centradas en la emoción (Piko, 2001; Renk y Creasey, 2003).

Finalmente conviene destacar alguna de las limitaciones de este estudio como es su naturaleza transversal, que impide realizar inferencias de causalidad y observar cambios en las estrategias de afrontamiento, por lo que sería interesante llevar a cabo estudios longitudinales en el futuro. Por otra parte, la recogida de datos a través de autoinforme implica la posibilidad de que las respuestas proporcionadas por los y las adolescentes podrían estar bajo la influencia de la deseabilidad social o de otros sesgos. Sin embargo, conviene destacar también, las aportaciones de este estudio al utilizar una muestra representativa de adolescentes escolarizados, así como la relevancia de los resultados hallados, especialmente en el caso de las adolescentes. 


\section{Bibliografía}

BERMÚDEZ, M.P.; TEVA, I. y BUELA-CASAL, G., "Influencia de variables sociodemográficas sobre los estilos de afrontamiento, el estrés social y la búsqueda de sensaciones sexuales en adolescentes". Psicothema, 21, (2) (2009), pp. 220-226.

DONENBERG, G.T. y PAO, M., "Psychiatry's role in a changing epidemic". Journal of the American Child and Adolescent Psychiatry 44 (2005), pp. 728-747.

FANSHAWE, J.P. y BURNETT, P.C., "Assessing school-related stressors and coping mechanisms in adolescents". British Journal of Educational Psychology 61 (1991), pp. 92-98.

FRYDENBERG, E., "Adolescent concerns: The concomitants of coping". Australian Journal of Educational and Developmental Psychology, 4 (1994), pp. $1-11$.

FRYDENBERG, E. y LEWIS, R., "Adolescent coping: The different ways in which boys and girls cope". Journal of Adolescence 14 (1991), pp. 119-133.

-, Manual: ACS. Escalas de afrontamiento para adolescentes. Madrid: TEA Ediciones, 1996.

-, "Things don't better just because you are older: A case for facilitating reflection". British Journal of Educational Psychology 69 (1999), pp. 81-94.

-, "Teaching coping to adolescents: when and whom?". American Educational Research Journal 37, (3) (2000), pp. 727-745.

GALAIF, E.R.; SUSSMAN, S.; CHIH-PING, C. y WILLS, A.T., "Longitudinal relations among depression, stress and coping in high risk youth". Journal of Youth and Adolescence 32 (2003), pp. 243-258.

GONZÁLEZ BARRÓN, R.; MONTOYA CASTILLA, I.; CASULLO, M.M. y BERNABEU VERDÚ, J., "Relación entre estilos y estrategias de afrontamiento y bienestar psicológico en adolescentes". Psicothema 14 (2002), pp. 363-368.

HAMPEL, P. y PETERMANN, F., "Perceived stress, coping and adjustment in adolescents". Journal of Adolescent Health 38 (2006), pp. 409-415.

LAZARUS, R.S. y FOLKMAN, S., Stress, appraisal and coping. New York: Springer Publising Company, 1984.

MULLIS, R.L. y CHAPMAN, P., "Age, gender and self-esteem differences in adolescent coping styles". Journal of Social Psychology 140 (2000), pp. 539-541.

PATTERSON, J.M. y McCUBBIN, H.I., "Adolescent coping style and behaviors: conceptualization and measurement". Journal of Adolescence 10 (1987), pp. 163-186.

PLANCHEREL, B. y BOLOGNINI, M., "Coping and mental health in early adolescence". Journal of Adolescence 18 (1995), pp. 459-474. 
PIKO, B., "Gender differences and similarities in adolescents' ways of coping". Psychological Record 51 (2001), pp. 223-235.

RECKLITIS, C.J. y NOAM, G.G., "Clinical and developmental perspectives on adolescent coping". Child Psychiatry and Human Development 30 (1999), pp. 87-101.

RENK, K. y CREASEY, G., "The relationship of gender, gender identity and coping strategies in late adolescents". Journal of Adolescence 26 (2003), pp. 159-168.

SPIRITO, A.; STARK, L.J. y WILLIAMS, C., "Development of brief coping checklist for use with pediatric populations". Journal of Pediatric Psychology 13 (1988), pp. 555-574.

WASHBURN, J.M.; HILLMAN, S.B. y SAWILOWSKY, S., "Gender and genderrole orientation differences on adolescents". Journal of Youth and Adolescence 33 (2004), pp. 31-40. 